\title{
TIME-SYMMETRIZED QUANTUM THEORY
}

\author{
Lev Vaidman \\ School of Physics and Astronomy \\ Raymond and Beverly Sackler Faculty of Exact Sciences \\ Tel Aviv University, Tel-Aviv 69978, Israel.
}

\begin{abstract}
A brief review of the time-symmetrized quantum formalism originated by Aharonov, Bergmann and Lebowitz is presented. Symmetry of various measurements under the time reversal is analyzed. Time-symmetrized counterfactuals are introduced. It is argued that the time-symmetrized formalism demonstrates novel profound features of quantum theory and that recent criticism of the formalism is unfounded.
\end{abstract}

\section{Introduction.}

The time-symmetrized quantum theory (TSQT) originated in a seminal work of Aharonov, Bergmann, and Lebowitz (ABL) [1]. Since then Aharonov and co-workers have developed a rich formalism 22 which has led to the discovery of numerous bizarre effects in quantum theory [3, 4, 5, 6, 77. Alternative time-symmetrized approaches have been suggested by other authors [8, 9,10$]$. Recently, however, the validity of some of the results of the TSQT, especially in the context of counterfactual interpretation of the ABL rule, have been questioned [11]. The purpose of this paper is to explain the meaning of the TSQT developed by Aharonov's group, to review time-symmetry properties in the framework of this formalism, to give a brief answer to the critics of the TSQT and to define time-symmetrized counterfactuals in quantum theory.

The novelty of the TSQT follows from the observation that in quantum theory, contrary to the classical physics, the future measurements might add information about the present of a system. In the standard approach, a quantum system at a given time $t$ is described completely by a quantum state defined by the results of measurements performed on the system in the past (relative to the time $t$ ). In the TSQT a quantum system at a given time $t$ is described by a two-state vector defined by the results of measurements performed on the system in the past and in the future (relative to the time $t$ ). Thus, while standard quantum theory deals with pre-selected systems, the TSQT analyzes pre- and post-selected systems.

The purpose of the description of a quantum system by quantum state or by two-state vector is to connect the class of preparations which lead to the same set of effects on other systems. In Section 2 I shall explain how to prepare a quantum system described by various descriptions. In section 3 I analyze the outcomes of ideal measurements performed on systems prepared in different ways. Section 4 is devoted 
to generalized ideal measurements recently introduced by Shimony [12]. Section 5 analyzes the counterfactual interpretation of the ABL rule. In section 6 I describe weak measurements 13]. Section 7 concludes the paper with a brief discussion of the connection between the TSQT and the many-worlds interpretation [14] of quantum theory.

\section{Preparation of a Quantum System.}

(a) Pre-selected quantum systems.

In standard quantum theory a complete description of a system at a given time $t$ is given by a forward evolving quantum state, see Fig. $1 a$ :

$$
|\Psi\rangle .
$$

In order to prepare the quantum state (11) we have to perform a complete measurement in the past time $t_{1}, t_{1}<t$, and obtain a specific outcome $A=a$ such that after the unitary evolution from $t_{1}$ to $t$ the system will be in the desired state:

$$
|\Psi\rangle=U\left(t_{1}, t\right)|A=a\rangle .
$$

(b) Pre- and post-selected quantum systems.

The basic description of a quantum system in the TSQT is given by a two-state vector, see Fig. $1 b$ :

$$
\langle\Phi|| \Psi\rangle .
$$

In order to prepare the two-state vector (3), in addition to the measurement $A=a$ at time $t_{1}$, we have also to perform a complete measurement at $t_{2}, t_{2}>t$, and obtain

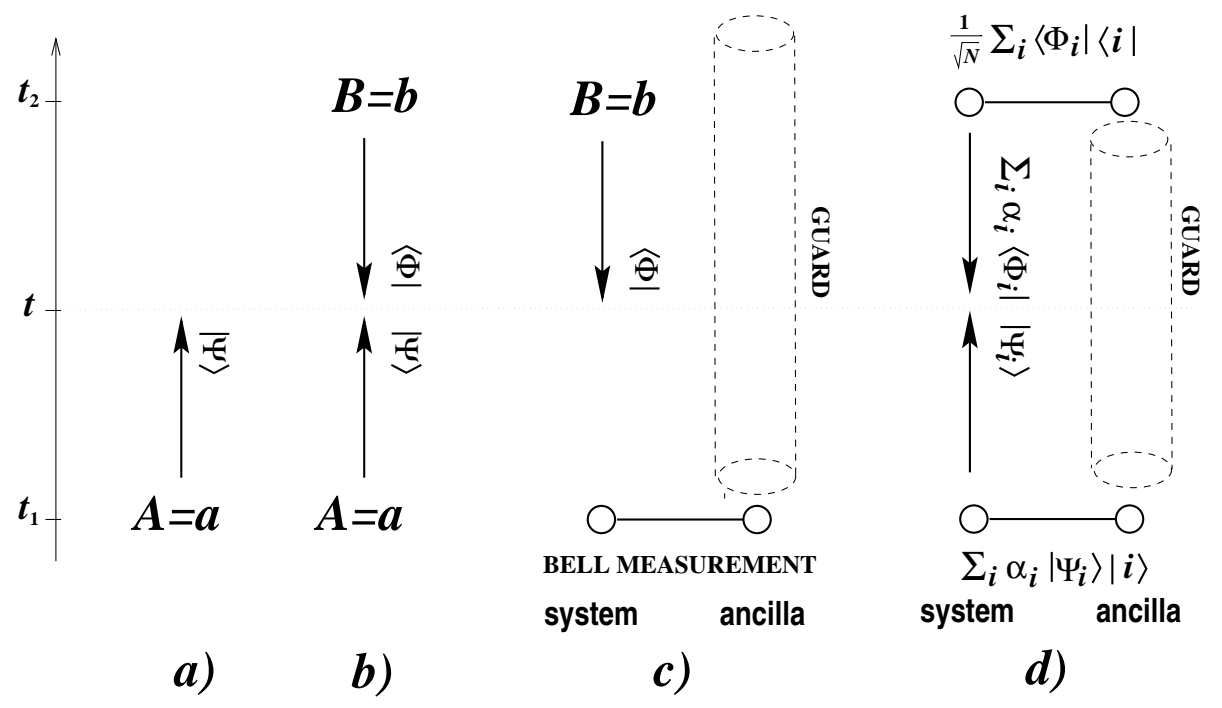

Fig. 1 Description of quantum systems: (a) pre-selected, (b) pre- and postselected, (c) post-selected, and (d) generalized pre- and post-selected. 
a specific outcome $B=b$ such that the backward time evolution from $t_{2}$ to $t$ will yield the desire state

$$
\langle\Phi|=\langle B=b| U\left(t_{2}, t\right) .
$$

In this presentation there is complete symmetry between preparation of the states $\langle\Phi|$ and $|\Psi\rangle$ which constitute the two-state vector: measurement of $A$ at $t_{1}$ leads to $|\Psi\rangle$ and the measurement of $B$ leads to $\langle\Phi|$. Both measurements might not yield the desired outcomes, so we need several systems out of which we pre- and post-select the one which is described by the two-state vector (3). However, this is only an apparent symmetry. There is an intrinsic difference in preparation of $|\Psi\rangle$ and $\langle\Phi|$. For preparation of $|\Psi\rangle$ a single system is enough. If the measurement of $A$ yields a different outcome $a^{\prime}$ we can perform a fast unitary operation which will change $\left|A=a^{\prime}\right\rangle$ to $|A=a\rangle$ and then the time evolution to time $t$ will bring the system to the state $|\Psi\rangle$. This procedure is impossible for preparation of the backward evolving state $\langle\Phi|$. Indeed, if the outcome of the measurement of $B$ does not yield $b$, we cannot read it and then make an appropriate unitary operation before $t_{2}$ in order to get the state $\langle\Phi|$ at time $t$. We need several systems to post-select the desired result (unless by chance the first system has the desired outcome).

In this paper I shall analyze symmetry under the interchange $\langle\Phi|| \Psi\rangle \leftrightarrow\langle\Psi|| \Phi\rangle$. This will be considered as a symmetry under reversal of the direction of the arrow of time. It is important to note that in general this interchange is not equivalent to the interchange of the measurements $A=a$ and $B=b$. (An example showing the non-equivalence can be found in the Appendix of Shimony's paper [12].) However, in order to simplify the discussion, I will assume that the free Hamiltonian is zero, and therefore $|\Psi\rangle=|A=a\rangle$ and $\langle\Phi|=\langle B=b|$. In this case, of course, the reversal of time arrow is identical to the interchange of the measurements at $t_{1}$ and $t_{2}$.

(c) Post-selected quantum systems.

I have presented above a description of quantum system by a single forwardevolving quantum state (11) and by a two-state vector (3). It is natural to ask: Are there systems described by a single backward-evolving quantum state? The notation for such a state is

$$
\langle\Phi| .
$$

A measurement of $B$ at time $t_{2}$, even in the case it yields the desired outcome $B=b$, is not enough. The difference between preparation of (11) and (5) is that at present, $t$, the future of a quantum system does not exist (the future measurements have not been performed yet), but the past of a quantum system exists: even if we do not know it, there is a quantum state of the system evolving towards the future defined by the results of measurements in the past. Therefore, in order to prepare a quantum system described by a backward evolving quantum state (5), in addition to the post-selection measurement performed after time $t$, we have to erase the past. (We do not have to perform a special erasure procedure for preparation of the pre- and post-selected system described by a two-state vector (3) because the complete measurement of $A$ at $t_{1}$ erases all prior information.)

In order to erase the past of a quantum system we can use another quantum system, an ancilla. The idea is to correlate an unknown future of the ancilla with the past of our system. For instance, such a correlation can be achieved using a Bell-type 
measurement, see Fig. 1c. For a spin-1/2 particle it can be realized by a sequence of two two-particle measurements [15]:

$$
\left(\sigma_{x}+\sigma_{x}^{(a n)}\right) \bmod 4, \quad\left(\sigma_{z}+\sigma_{z}^{(a n)}\right) \bmod 4 .
$$

Each measurement has two possible outcomes, 0 or 2. The four possible combinations correspond to four possible Bell state. Each Bell state yields complete correlation between the states of the two systems. A generalization to a system with continuous degrees of freedom can be done similarly to the generalization of teleportation to such systems [16]. The erasure procedure has to be supplemented by "guarding the ancilla" against any possible measurements after the Bell-type measurement. This will ensure that the past of the system will be correlated to the unknown future of the ancilla.

(d) Generalized pre- and post-selected quantum systems.

The technique of "guarded ancilla" is also used to create a quantum system described by a generalized two-state vector

$$
\sum_{i} \alpha_{i}\left\langle\Phi_{i}|| \Psi_{i}\right\rangle
$$

In order to prepare the generalized two-state vector (7) we have to prepare at $t_{1}$ the system and the ancilla in a correlated state $\sum_{i} \alpha_{i}\left|\Psi_{i}\right\rangle|i\rangle$, where $\{|i\rangle\}$ is a set of orthonormal states of the ancilla. Then we have to "guard" the ancilla such that there will be no measurements or any other interactions performed on the ancilla until the post-selection measurement of a projection on the correlated state $1 / \sqrt{N} \sum_{i}\left|\Phi_{i}\right\rangle|i\rangle$, see Fig. $1 d$. If we obtain the desired outcome then the system is described at time $t$ by the generalized two-state vector (7).

In the standard quantum theory the most general description of a quantum system is given by a mixed state or density matrix. For example, a quantum system after the "erasing the past" procedure proposed above is described by a density matrix and cannot be described by a pure state. It is believed, however, that there is always a larger system (which includes the quantum system) in a pure quantum state. (In particular, the composite system including the system with "erased past" and the ancilla which was used in the erasure procedure is in a pure state.) There is certain similarity between this situation and a system described by a generalized two-state vector: the composite system which includes the ancilla is described by a two-state vector (3). However, the analogy is not exact. A generalized two-state vector is not the most general description of a quantum system in the TSQT formalism - it is the most general complete description. It is possible to define the generalization of a twostate vector to a mixed case when various two-state vectors are correlated to another ancilla (wich is not post-selected). Although the generalization is straightforward, it is not obvious what is its most convenient form. For a powerful, but somewhat cumbersome formalism see Ref. [17].

\section{Ideal Quantum Measurements.}

In this section I shall discuss how a quantum system characterized by a certain description interacts with other systems. Some particular types of interactions are 
named measurements and the effect of these interactions characterized as the results of these measurements. The basic concept is an ideal quantum measurement of a an observable $C$. This operation is defined for pre-selected quantum systems in the following way:

(i) If the state of a quantum system before the measurement was an eigenstate of $C$ with an eigenvalue $c_{n}$ then the outcome of the measurement is $c_{n}$ and the quantum state of the system is not changed.

The standard implementation of the ideal quantum measurement is modeled by the von Neumann Hamiltonian [18:

$$
H=g(t) p C
$$

where $p$ is the momentum conjugate to the pointer variable $q$, and the normalized coupling function $g(t)$ specifies the time of the measurement interaction. The outcome of the measurement is the shift of the pointer variable during the interaction. In the ideal measurement the function $g(t)$ is nonzero only during a very short period of time, and the free Hamiltonian during this period of time can be neglected.

For a quantum system described by the two-state vector (3) the probability for an outcome $c_{n}$ of an ideal measurement of an observable $C$ is given by [1] 2

$$
\operatorname{Prob}\left(c_{n}\right)=\frac{\left|\left\langle\Phi\left|\mathbf{P}_{C=c_{n}}\right| \Psi\right\rangle\right|^{2}}{\sum_{j}\left|\left\langle\Phi\left|\mathbf{P}_{C=c_{j}}\right| \Psi\right\rangle\right|^{2}} .
$$

This formula is explicitly time-symmetric: First, both $\langle\Phi|$ and $|\Psi\rangle$ enter the equation on equal footing. Second, the probability (9) is unchanged under the interchange $\langle\Phi|| \Psi\rangle \leftrightarrow\langle\Psi|| \Phi\rangle$

For a quantum system described by a generalized two-state vector (7) the probability for an outcome $c_{n}$ is given by [2]

$$
\operatorname{Prob}\left(c_{n}\right)=\frac{\left|\sum_{i} \alpha_{i}\left\langle\Phi_{i}\left|\mathbf{P}_{C=c_{n}}\right| \Psi_{i}\right\rangle\right|^{2}}{\sum_{j}\left|\sum_{i} \alpha_{i}\left\langle\Phi_{i}\left|\mathbf{P}_{C=c_{j}}\right| \Psi_{i}\right\rangle\right|^{2}} .
$$

This formula is also time-symmetric. Indeed, $\left\langle\Phi_{i}\right|$ and $\left|\Psi_{i}\right\rangle$ enter the equation on equal footing. The manifestation of the symmetry of this formula under the reversal of the arrow of time includes complex conjugation of the coefficients. The probability (10) is unchanged under the interchange $\sum_{i} \alpha_{i}\left\langle\Phi_{i}|| \Psi_{i}\right\rangle \leftrightarrow \sum_{i} \alpha_{i}^{*}\left\langle\Psi_{i}|| \Phi_{i}\right\rangle$.

Another important generalization of the formula (9) is for the case in which the post-selection measurement is not complete and therefore it does not specify a single post-selection state $\langle\Phi|$. Such an example was recently considered by Cohen [19] in (an unsuccessful [20]) attempt to find constraints to the applicability of the ABL formula. In this case, the post-section measurement is a projection on a degenerate eigenvalue of an observable $B=b$. The modified ABL formula is 20]:

$$
\operatorname{Prob}\left(c_{n}\right)=\frac{\| \mathbf{P}_{B=b} \mathbf{P}_{C=c_{n}}|\Psi\rangle \|^{2}}{\sum_{j} \| \mathbf{P}_{B=b} \mathbf{P}_{C=c_{j}}|\Psi\rangle \|^{2}} .
$$


This form of the ABL formula allows to connect it to the standard formalism of quantum theory in which there is no post-selection. In the limiting case when the projection operator $\mathbf{P}_{B=b}$ is just unity operator $\mathbf{I}$, we obtain the usual expression:

$$
\operatorname{Prob}\left(c_{n}\right)=\| \mathbf{P}_{C=c_{n}}|\Psi\rangle \|^{2} .
$$

\section{Generalized Ideal Quantum Measurements.}

Recently, Shimony [12] proposed the following generalization of the concept of ideal measurements by weakening the requirement given in definition (i):

(ii) If the state of a quantum system before the measurement is an eigenstate of $C$ with an eigenvalue $c_{n}$ then the outcome of the measurement is $c_{n}$ and the quantum state of the system after the measurement remains in the subspace of the eigenstates corresponding to this eigenvalue.

The difference between definitions (i) and (ii) is that while in the ideal measurements the change of the state during the measurement happens only if the initial state was a superposition of states with different eigenvalues, the generalized ideal measurement procedure changes also the eigenstates themselves. (This might happen only for degenerate eigenvalues.) Shimony has constructed a generalized ideal measurement for which the ABL formula (9) does not hold. I shall derive Shimony's result using another example.

Consider a composite system of two spin- $\frac{1}{2}$ particles. The variable to be measured is the value of the total spin of the system which can be 0 or 1 . The total spin 0 corresponds to a nondegenerate state (singlet), while total spin 1 corresponds to three orthogonal eigenstates (triplet). The generalized ideal measurement do not change measured value (the total spin), but it might change a commuting variable. In this example the measurement changes cyclicly the total $\hat{z}$ component of the spin (for triplet). This measurement is described by the following unitary transformation:

$$
\begin{aligned}
|0,0\rangle|R\rangle_{M D} & \rightarrow|0,0\rangle|0\rangle_{M D} \\
|1,-1\rangle|R\rangle_{M D} & \rightarrow|1,0\rangle|1\rangle_{M D} \\
|1,0\rangle|R\rangle_{M D} & \rightarrow|1,1\rangle|1\rangle_{M D} \\
|1,1\rangle|R\rangle_{M D} & \rightarrow|1,-1\rangle|1\rangle_{M D}
\end{aligned}
$$

where $|0,0\rangle,|1,-1\rangle$ etc. denote the states of the composite system in $S^{2}, S_{z}$ representation, while $|R\rangle_{M D},|0\rangle_{M D}$ and $|1\rangle_{M D}$ denote the "ready" and "0" and "1" final states of the measuring device.

Now consider the measurement of the total spin performed on a system pre- and post-selected in a two-state vector

$$
\langle\Phi|| \Psi\rangle=\frac{1}{2}(\langle 0,0|+\langle 1,1|)(|0,0\rangle+|1,0\rangle) .
$$

Given the pre-selection, $|\Psi\rangle=1 / \sqrt{2}(|0,0\rangle+|1,0\rangle)$, only, the probability for the two possible outcomes are equal:

$$
\operatorname{Prob}(0 ;|\Psi\rangle)=\operatorname{Prob}(1 ;|\Psi\rangle)=\frac{1}{2} .
$$


If the outcome is " 0 " then, after the measurement, the state of the system is $|0,0\rangle$; if, the outcome is " 1 " then the final state is $|1,1\rangle$. It turns out that in both cases the probability for the post-selection of the state, $\langle\Phi|=1 / \sqrt{2}(\langle 0,0|+\langle 1,1|)$, is:

$$
\operatorname{Prob}(\langle\Phi| ; 0)=\operatorname{Prob}(\langle\Phi| ; 1)=\frac{1}{2} .
$$

Then, Bayes theorem yields the probability of obtaining the outcome "1" in the generalized measurement performed on the pre- and post-selected system:

$$
\operatorname{Prob}(1 ;\langle\Phi|| \Psi\rangle)=\frac{\operatorname{Prob}(1 ;|\Psi\rangle) \operatorname{Prob}(\langle\Phi| ; 1)}{\operatorname{Prob}(0 ;|\Psi\rangle) \operatorname{Prob}(\langle\Phi| ; 0)+\operatorname{Prob}(1 ;|\Psi\rangle) \operatorname{Prob}(\langle\Phi| ; 1)}=\frac{1}{2}
$$

Let us repeat the calculation for the time reversed two-state vector:

$$
\langle\Psi|| \Phi\rangle=\frac{1}{2}(\langle 0,0|+\langle 1,0|)(|0,0\rangle+|1,1\rangle) .
$$

Now, after the generalized measurement with outcome "1", the state of the system must be $|1,-1\rangle$. This state, however, is orthogonal to the post-selected state and therefore this outcome is impossible. Thus, $\operatorname{Prob}(\langle\Psi| ; 1)=0$ and consequently, $\operatorname{Prob}(1 ;\langle\Psi|| \Phi\rangle)=0$.

I have shown that in this example there is an asymmetry: $\operatorname{Prob}(1 ;\langle\Phi|| \Psi\rangle) \neq$ $\operatorname{Prob}(1 ;\langle\Psi|| \Phi\rangle)$. Therefore, the ABL formula, which is symmetric under such interchange, cannot hold. (It yields $\operatorname{Prob}(1 ;\langle\Phi|| \Psi\rangle)=\operatorname{Prob}(1 ;\langle\Psi|| \Phi\rangle)=0$.) I find this result not too disturbing since these measurements have an unusual property even in a situation in which the ABL formula is not involved: generalized ideal measurements of commuting observables might disturb each other.

\section{Counterfactual Interpretation of the ABL Rule.}

Several authors criticized the TSQT because of the alleged conflict between counterfactual interpretations of the ABL rule and predictions of quantum theory 11, 19, 21. The form of all these inconsistency proofs is as follows: The probability of an outcome $C=c_{n}$ of a quantum measurement performed on a pre-selected system, given correctly by (12), is considered. In order to allow the analysis using the ABL formula, a measurement at a later time, $t_{2}$, with two possible outcomes, which we denote by " $1_{f}$ " and " $2_{f}$ ", is introduced. The suggested application of the ABL rule is expressed in the formula for the probability of the result $C=c_{n}$ :

$$
\operatorname{Prob}\left(C=c_{n}\right)=\operatorname{Prob}\left(1_{f}\right) \operatorname{Prob}\left(C=c_{n} ; 1_{f}\right)+\operatorname{Prob}\left(2_{f}\right) \operatorname{Prob}\left(C=c_{n} ; 2_{f}\right),
$$

where $\operatorname{Prob}\left(C=c_{n} ; 1_{f}\right)$ and $\operatorname{Prob}\left(C=c_{n} ; 2_{f}\right)$ are the conditional probabilities given by the ABL formula, (9), and $\operatorname{Prob}\left(1_{f}\right)$ and $\operatorname{Prob}\left(2_{f}\right)$ are the probabilities of the results of the final measurement. In the proofs, the authors show that Eq. (19) is not valid and conclude that the ABL formula is not applicable to this example and therefore it is not applicable in general.

I have argued [22, 23, 20] that the error in calculating equality (19) does not arise from the conditional probabilities given by the ABL formula, but from the 
calculation of the probabilities $\operatorname{Prob}\left(1_{f}\right)$ and $\operatorname{Prob}\left(2_{f}\right)$ of the final measurement. In all the three alleged proofs the probabilities $\operatorname{Prob}\left(1_{f}\right)$ and $\operatorname{Prob}\left(2_{f}\right)$ were calculated on the assumption that no measurement took place at time $t$. Clearly, one cannot make this assumption here since then the discussion about the probability of the result of the measurement at time $t$ is meaningless. Thus, it is not surprising that the value of the probability $\operatorname{Prob}\left(C=c_{n}\right)$ obtained in this way comes out different from the value predicted by the quantum theory. Straightforward calculations show that the formula (19) with the probabilities $\operatorname{Prob}\left(1_{f}\right)$ and $\operatorname{Prob}\left(2_{f}\right)$ calculated on the condition that the intermediate measurement has been performed leads to the result predicted by the standard formalism of quantum theory.

The analysis of counterfactual statements considers both actual and counterfactual worlds. The statement is considered to be true if it is true in counterfactual worlds "closest" to the actual world. In the context of the ABL formula, in the actual world the pre-selection and the post-selection has been successfully performed, but the measurement of $C$ has not (necessarily) been performed. On the other hand, in counterfactual worlds the measurement of $C$ has been performed. The problem is to find counterfactual worlds "closest" to the actual world in which the measurement of $C$ has been performed. The fallacy in all the inconsistency proofs is that their authors have considered counterfactual worlds in which $C$ has not been measured.

Even if we disregard this fallacy there is still a difficulty in defining the "closest" worlds in the framework of the TSQT. In standard quantum theory it is possible to use the most natural definition of the "closest" world. Since the future is considered to be irrelevant for measurements at present, $t$, only the period of time before $t$ is considered. Then the definition is:

(iii) Closest counterfactual worlds are the worlds in which the system is described by the same quantum state as in the actual world.

In the framework of the TSQT, however, this definition is not acceptable. In the time-symmetric approach the period of time before and after $t$ is considered. The measurement of $C$ constrains the possible states immediately after $t$ to the eigenstates of $C$. Therefore, if in the actual world the state immediately after $t$ is not an eigenstate of $C$, no counterfactual world with the same state exists. Moreover, there is the same problem with the backward evolving quantum state (the concept which does not exist in the standard approach) in the period of time before $t$. I proposed [23] to solve this difficulty by adopting the following definition of the closest world:

(iv) Closest counterfactual worlds are the worlds in which the results of all measurements performed on the system (except the measurement at time $t$ ) are the same as in actual world.

For the pre-selected only situation, this definition is equivalent to (iii), but it is also applicable to the symmetric pre- and post-selected situation.

An important example of counterfactuals in quantum theory are "elements of reality". I have proposed a modification of the definition of elements of reality applicable to the framework of the TSQT [24]: 
(vi) If we can infer with certainty that the result of measuring at time $t$ of an observable $C$ is $c$, then, at time $t$, there exists an element of reality $C=c$.

The word "infer" is neutral relative to past and future. The inference about results at time $t$ is based on the results of measurements on the system performed both before and after time $t$. Note, that there are situations (see example with a particle in three boxes below) in which we can "infer" some facts that cannot be obtained by neither "prediction" based on the past results nor "retrodiction" based on the future results separately.

\section{Weak Measurements.}

The most interesting phenomena which can be seen in the framework of the TSQT are related to weak measurements [13]. A weak measurement is a standard measuring procedure (described by the Hamiltonian (8) with weakened coupling. In ideal measurement the initial position of the pointer $q$ is well localized around zero and therefore the conjugate momentum $p$ has a very large uncertainty which leads to a very large uncertain Hamiltonian of the measurement (8). In weak measurement, the initial state of the measuring device is such that $p$ is localized around zero with small uncertainty. This leads, of course, to a large uncertainty in $q$ and therefore the measurement becomes imprecise. However, by performing the weak measurement on an ensemble of $N$ identical systems we improve the precision by the factor of $\sqrt{N}$ and in some special cases we can obtain good precision even in a measurement performed on a single system [3]. The outcome of a weak measurement of a variable $C$ is the weak value. The weak value of a variable $C$ of a system described by the two-state vector $\langle\Phi|| \Psi\rangle$ is:

$$
C_{w} \equiv \frac{\langle\Phi|C| \Psi\rangle}{\langle\Phi \mid \Psi\rangle}
$$

Strictly speaking, the readings of the pointer of the measuring device will cluster around $\operatorname{Re}\left(C_{w}\right)$. In order to find $\operatorname{Im}\left(C_{w}\right)$ one should measure the shift in $p$ [13].

The weak value is symmetric under the interchange $\langle\Phi|| \Psi\rangle \leftrightarrow\langle\Psi|| \Phi\rangle$ provided we perform complex conjugation of the weak value together with the interchange. This is similar to complex conjugation of the Schrödinger wave function under the time reversal. Thus, also for weak measurement there is time reversal symmetry: both $\langle\Phi|$ and $|\Psi\rangle$ enter the formula of the weak value on the same footing and there is symmetry under the interchange of the pre- and post-selected states.

The weak value for a system described by a generalized two-state vector (7) is [2]:

$$
C_{w}=\frac{\sum_{i} \alpha_{i}\left\langle\Phi_{i}|C| \Psi_{i}\right\rangle}{\sum_{i} \alpha_{i}\left\langle\Phi_{i} \mid \Psi_{i}\right\rangle} .
$$

This expression is also symmetric under the time reversal, i.e., the interchange $\sum_{i} \alpha_{i}\left\langle\Phi_{i}|| \Psi_{i}\right\rangle \leftrightarrow \sum_{i} \alpha_{i}^{*}\left\langle\Psi_{i}|| \Phi_{i}\right\rangle$ leads to $C_{w} \leftrightarrow C_{w}^{*}$.

Next, let us look at the expression for the weak value when the post-selection measurement is not complete. Consider a system pre-selected in the state $|\Psi\rangle$ and 
post-selected by the the measurement of degenerate eigenvalue $b$ of a variable $B$. (The ABL formula for the probability of $C=c_{n}$ for such situation is given by (11).

$$
C_{w}=\frac{\left\langle\Psi\left|\mathbf{P}_{B=b} C\right| \Psi\right\rangle}{\left\langle\Psi \mathbf{P}_{B=b} \mid \Psi\right\rangle} .
$$

This formula allows us to find the outcome of a weak measurement performed on a pre-selected (only) system. Replacing $\mathbf{P}_{B=b}$ by the unity operator yields the result that the weak value of a pre-selected system in the state $|\Psi\rangle$ is the expectation value:

$$
C_{w}=\langle\Psi|C| \Psi\rangle
$$

Weak values have many interesting properties, in particular, $(A+B)_{w}=A_{w}+B_{w}$, even for non-commuting observables $A$ and $B$. I have considered weak values as weak-measurement of reality [25], but the weak value is not just a theoretical concept related to a gedanken experiment. Recently, weak values have been measured in a real laboratory [26].

An interesting connection between weak and strong (ideal) measurements is a theorem [2] which says that if the probability for a certain value to be the result of a strong measurement is 1 , then the corresponding weak measurement must also yield the same value, i.e., element of reality $C=c$, implies weak-measurement element of reality $C_{w}=c$. Consider a single particle located in three separated boxes $A, B$, and $C$ pre- and post- selected in the two-state vector

$$
\langle\Phi|| \Psi\rangle=\frac{1}{3}(\langle A|+\langle B|-\langle C|)(|A\rangle+|B\rangle+|C\rangle) .
$$

A set of counterfactual statements for this particle is:

$$
\mathbf{P}_{A}=1, \quad \mathbf{P}_{B}=1, \quad \mathbf{P}_{A}+\mathbf{P}_{B}+\mathbf{P}_{C}=1
$$

Or, in words: if we open box $A$, we find the particle there for sure; if we open box $B$ (instead), we also find the particle there for sure; if we open simultaneously all boxes, we find the particle in one of them for sure. These counterfactual statements lead us to statements about weak-measurement elements of reality:

$$
\left(\mathbf{P}_{A}\right)_{w}=1, \quad\left(\mathbf{P}_{B}\right)_{w}=1, \quad\left(\mathbf{P}_{A}+\mathbf{P}_{B}+\mathbf{P}_{C}\right)_{w}=1
$$

From these results we can also deduce that $\left(\mathbf{P}_{C}\right)_{w}=-1$. Therefore, for every sufficiently weak interaction the effective coupling to this single particle is equivalent to the weak coupling to a single particle in box $A$, a single particle in box $B$ and minus one particle in box $C$. The meaning of the latter is that for a pre- and post-selected ensemble of many such particles there is an effective negative pressure in box $C$. However, an experiment which will test this is very difficult because the probability to obtain such an ensemble is very low.

\section{Conclusions.}

In this paper I presented basic concepts of the TSQT. In order to get a complete picture one should read also other works which were cited here, but some topics were 
analyzed in this paper more completely than in other publications. Among them the issue of preparation of a quantum system described in a time-symmetrized way. Here "pre" of the word preparation is somewhat misleading, but I could not find more appropriate word. The absence of a word in English which describes exactly the situation follows from the fact that until very recently we had no reason to believe that the result of future measurements are relevant to the discussion of a measurement at present. In this context I want to mention that the time-symmetrized quantum theory fits well into the many-worlds interpretation (MWI) [14, my preferred interpretation of quantum theory [27]. The counterfactual worlds corresponding to different outcomes of quantum measurements have in the MWI an especially clear meaning: these are subjectively actual different worlds. In each world the observers of the quantum measurement call their world the actual one, but, if they believe in the MWI they have no paradoxes about ontology of the other worlds. The apparent paradox that a weak value at a given time might change from an expectation value to a weak value corresponding to a particular post-selection is solved in a natural way: in a world with pre-selection only (before the post-selection) the weak value is the expectation value; then this world splits into several worlds according to results of the post-selection measurement and in each of these worlds the weak value will be that corresponding to the particular post-selection.

Another issue which was elaborated here in detail is the time-symmetry of the generalized ideal measurements recently proposed by Shimony. I showed explicitly why these measurements are not symmetric under the time reversal.

An important novel issue which was briefly considered here is time-symmetrized counterfactuals in quantum theory. These results have not been published yet in other journals, but one can read more on this subject in the preprints [20, 22, 23].

The most important issue in the framework of the TSQT, the issue of weak measurements, was also discussed here only briefly and I recommend reading the review lecture [28]. The connection to protective measurements [29] was not covered at all. The only novel point related to weak measurements in this work is Eq. (22) which yields the weak value in the case of partial post-selection.

It is a pleasure to thank Yakir Aharonov, Jacob Grunhaus and Stephen Wiesner for helpful discussions. The research was supported in part by grant $614 / 95$ of the

Basic Research Foundation (administered by the Israel Academy of Sciences and Humanities).

\section{References}

[1] Y. Aharonov, P. G. Bergmann and J. L. Lebowitz, Phys. Rev. B134, 1410 (1964).

[2] Y. Aharonov and L. Vaidman, J. Phys. A 24, 2315 (1991).

[3] Y. Aharonov, D. Albert, A. Casher, and L. Vaidman, Phys. Lett. A 124, 199 (1987).

[4] L. Vaidman, Y. Aharonov and D. Albert, Phys. Rev. Lett. 58, 1385 (1987).

[5] Y. Aharonov, D. Albert, and L. Vaidman, Phys. Rev. Lett. 60, 1351 (1988). 
[6] Y. Aharonov, J. Anandan, and L. Vaidman, Phys. Rev. A 47, 4616 (1993).

[7] Y. Aharonov, S. Popescu, D. Rohrlich, and L. Vaidman, Phys. Rev. A 48, 4084 (1993).

[8] Rev. Mod. Phys. 58, 647 (1986).

[9] R. B. Griffiths, J. Stat. Phys. 36, 219 (1984).

[10] M. Gell-Mann and J. B. Hartle, in W. H. Zurek (ed), Complexity, Entropy and the Physics of Information, (Reading: Edison-Wesley), pp. 425-459 (1990).

[11] W. D. Sharp and N. Shanks, Phil. Sci. 60, 488 (1993).

[12] A. Shimony, Erken. 45, 337 (1997).

[13] Y. Aharonov and L. Vaidman, Phys. Rev. A 41, 11 (1990).

[14] H. Everett, Rev. Mod. Phys. 29, 454 (1957).

[15] Y. Aharonov, D. Albert, and L. Vaidman, Phys. Rev. D 34, 1805 (1986).

[16] L. Vaidman, Phys. Rev. A 49, 1473 (1994).

[17] B. Reznik and Y. Aharonov, Phys. Rev. A, 52, 2538 (1995).

[18] J. von Neumann, Mathematical Foundations of Quantum Theory, (Princeton, University Press, New Jersey (1983).

[19] O. Cohen. (1995), Phys. Rev. A 51, 4373 (1995).

[20] L. Vaidman, Tel-Aviv University preprint quant-ph/9703001.

[21] D. J. Miller, Phys. Lett. A 222, 31 (1996).

[22] L. Vaidman, Tel-Aviv University preprint quant-ph/9609007.

[23] L. Vaidman, Tel-Aviv University preprint TAUP 2459-97.

[24] L. Vaidman, Phys. Rev. Lett. 70: 3369(1993).

[25] L. Vaidman, Found. Phys. 26, 895 (1996).

[26] N.W.M. Ritchie, J.G. Story, and R.G. Hulet, Phys Rev. Lett. 66, 1107 (1991).

[27] L. Vaidman, preprint quant-ph/9609006, to be published in Int. Stud. Phil. Sci.

[28] L. Vaidman, in Advances in Quantum Phenomena, E. Beltrametti and J.M. LevyLeblond eds., NATO ASI Series B: Physics Vol. 347, Plenum Press, NY, pp. 357-373 (1995).

[29] Y. Aharonov and L. Vaidman, in Potentiality, Entanglement and Passion-at-aDistance, R.S.Cohen, et al. (eds), Kluwer Academic Publishers, pp.1-8, (1997). 